1 Hacettepe Journal of Mathematics and Statistics

$\bigcap$ Volume $44(4)(2015), 801-811$

\title{
Quasi-primary submodules satisfying the primeful property II
}

\author{
Hosein Fazaeli Moghimi * and Mahdi Samiei ${ }^{\dagger}$
}

\begin{abstract}
In this paper we continue our study about quasi-primary submodules (probably satisfying the primeful property), that was defined and studied in Part I (see [8]). We define a quasi-primary decomposition for submodules of a module over a commutative ring with identity and study various types of the corresponding minimal forms. In particular, we discuss these decompositions for submodules of multiplication modules and also arbitrary modules over Noetherian rings.
\end{abstract}

2000 AMS Classification: 13C13, 13C99.

Keywords: Quasi-primary submodule, Primeful property, Radical of a submodule, Multiplication module, Reduced quasi-primary decomposition, Modulereduced quasi-primary decomposition, Shortest quasi-primary decomposition.

Received 28/01/2013 : Accepted 14/04/2014 Doi : 10.15672 /HJMS.2015449436

\section{INTRODUCTION}

Throughout this paper all rings are commutative with identity, and all modules are unitary. Recently, the decomposition theory associated with various generalizations of prime and primary ideals has been the domain of concerns of many researches (see for example $[18,21,24])$. Here we follow this topic in the context of quasi-primary submodules; the recent generalization of quasi-primary ideals. Some concepts which are used frequently in this paper have been gathered in the following definition.

1.1. Definition. Let $N$ be a proper submodule of an $R$-module $M$.

(1) $N$ is prime(resp. primary) if $r x \in N$ for $r \in R$ and $x \in M$ implies either $r \in(N: M)($ resp. $r \in \sqrt{(N: M)})$ or $x \in N$ (see [5, 14, 22, 15, 17]).

(2) The intersection of all prime submodules of $M$ containing $N$, denoted $\operatorname{rad} N$, is called prime radical of $N$ (see $[3,10,13,16,19,26])$.

${ }^{*}$ Department of Mathematics, University of Birjand, P.O.Box 97175-615, Birjand, Iran. Email: hfazaeli@birjand.ac.ir

${ }^{\dagger}$ Department of Mathematics, University of Birjand, P.O.Box 97175-615, Birjand, Iran. Email:mahdisamiei@birjand.ac.ir 
(3) $N$ is quasi-primary if $r x \in N$ for $r \in R$ and $x \in M$, then either $r \in \sqrt{(N: M)}$ or $x \in \operatorname{rad} N$. Clearly every primary submodule is quasi-primary, but not conversely in general (see Example 1.2 and Example 2.3).

(4) $N$ satisfies the primeful property provided that for every prime ideal $p$ containing $(N: M)$ there exists a prime submodule $P$ contains $N$ such that $(P: M)=p$. In particular, $M$ is primeful if the zero submodule of $M$ satisfies the primeful property. Every submodule of a finitely generated module satisfies the primeful property (see $[8,12])$.

(5) $N$ has a quasi-primary decomposition if $N=N_{1} \cap N_{2} \cap \cdots \cap N_{s}$, where each $N_{i}$ is a quasi-primary submodule of $M$. If $N_{i} \nsupseteq N_{1} \cap \cdots \cap N_{i-1} \cap N_{i+1} \cap \cdots \cap N_{s}$, then the above quasi-primary decomposition is called

(5.1) reduced, if the ideals $\sqrt{\left(N_{i}: M\right)}$ are distinct primes.

(5.2) module-reduced, if the submodules $\operatorname{rad} N_{i}$ are distinct primes.

(5.3) shortest, if none of the intersection $\left(N_{i_{1}}: M\right) \cap\left(N_{i_{2}}: M\right) \cap \cdots \cap\left(N_{i_{t}}: M\right)$ $(t>1)$ is a quasi-primary ideal.

(6) An $R$-module $M$ is said to be a multiplication module, if every submodule of $M$ has the form $I M$ for some ideal $I$ of $R$. For example any cyclic module is a multiplication module. However, there is a multiplication module which is not finitely generated $[7$, p.770]. Also, free modules with finite rank greater than one are finitely generated modules which are not multiplication modules $[15$, Corollary 2.5 and Theorem 3.5]. It is well-known that $M$ is a multiplication $R$-module if and only if for each submodule $N$ of $M, N=(N: M) M$. (see for more study $[1,7,23])$.

(7) The support of M, written $\operatorname{Supp}(M)$, is defined to be the set of prime ideals $p$ of $R$ such that $M_{p} \neq 0$ (see $[6,20]$ ).

(8) A prime ideal $p$ of $R$ is associated to $M$ if $p$ is the annihilator of an element of $M$. The set of all primes associated to $M$ is denoted by $A s s(M)$ (see $[6,20]$ ).

1.2. Example. Indeed, every power of a prime ideal as well as that of a primary or a quasi-primary ideal is quasi-primary; but a power of a prime ideal is not necessarily primary (for example see [2, Example after proposition 4.1, part 3]). Now we follow this fact to give an example in the module setting. It is well-known that if $F$ is a free $R$-module and $I$ is an ideal of $R$, then $(I F: F)=I$ and $\operatorname{rad}(I F)=\sqrt{I} F[25$, Proposition 2.2 . It is routine to verify that $q$ is a quasi-primary (resp. primary, prime) ideal of $R$ if and only if $q F$ is a quasi-primary (resp. primary, prime) submodule of $F$ [8, Theorem 2.19]. These show that there is a rich supply of quasi-primary submodules which are not primary.

Recall that a proper ideal $q$ of $R$ is quasi-primary if $r s \in q$ for $r, s \in R$ implies $r \in \sqrt{q}$ or $s \in \sqrt{q}$ (see [8,9]). It is well-known that $q$ is a quasi-primary ideal of $R$ if and only if $\sqrt{q}$ is a prime ideal of $R$ [9, p.176]. For a submodule $N$ of a multiplication $R$-module $M$ which satisfies the primeful property, we prove that $N$ is a quasi-primary submodule of $M$ if and only if $(N: M)$ is a quasi-primary ideal of $R$ if and only if $\operatorname{radN}$ is a prime submodule of $M$ if and only if $N=q M$ for some quasi-primary ideal $q$ of $R$ with $\operatorname{ann}(M) \subseteq q$ (Theorem 2.2). We use this fact to investigate the relationships between reduced and module-reduced and shortest quasi-primary decompositions of submodules of multiplication modules (Corollary 2.6 and Proposition 2.11 and Theorem 2.13). Also we give some uniqueness theorems as follow:

Theorem 2.13. Let $M$ be a multiplication $R$-module and $N$ a submodule of $M$. Let $N=N_{1} \cap N_{2} \cap \cdots \cap N_{s}=N_{1}^{\prime} \cap N_{2}^{\prime} \cap \cdots \cap N_{t}^{\prime}$ be two reduced quasi-primary decompositions of $N$ as intersection of quasi-primary submodules satisfying the primeful property. Then $s=t$ and the prime ideals $p_{i}=\sqrt{\left(N_{i}: M\right)}$ must be, without regard to their order, 
identical to the prime ideals $p_{j}^{\prime}=\sqrt{\left(N_{j}^{\prime}: M\right)}$.

Theorem 3.5. Let $R$ be a Noetherian ring and $M$ an $R$-module. Let $N$ be a submodule of $M$ such that $N=N_{1} \cap N_{2} \cap \cdots \cap N_{s}=N_{1}^{\prime} \cap N_{2}^{\prime} \cap \cdots \cap N_{t}^{\prime}$ be two reduced quasi-primary decompositions of $N$ where $N_{i}$ (resp. $N_{j}^{\prime}$ ) is $\mathrm{p}_{i}$-quasi-primary (resp. $\mathfrak{p}_{j}$-quasi-primary). Then $s=t$ and (after reordering if necessary) $\mathrm{p}_{i}=\mathfrak{p}_{i}$ and $\operatorname{rad} N_{i}=\operatorname{rad} N_{i}^{\prime}$ for $1 \leq i \leq s$. Theorem 3.7. Let $N$ be a proper submodule of a module $M$ over a Noetherian ring $R$. If $N=\cap_{i=1}^{s} N_{i}$ is a module-reduced quasi-primary decomposition and $N_{i}(1 \leq i \leq s)$ satisfies the primeful property such that $\operatorname{rad} N=\cap_{i=1}^{s} \operatorname{rad} N_{i}$, then $A s s(M / \operatorname{rad} N) \subseteq$ $\left\{p_{1}, \cdots, p_{s}\right\} \subseteq \operatorname{Supp}(M / \operatorname{rad} N)$. In particular, $\operatorname{Ass}(M / \operatorname{rad} N)=\left\{p_{i_{1}}, p_{i_{2}}, \cdots, p_{i_{t}}\right\}$ where $p_{i_{j}} 1 \leq j \leq t$ are minimal elements of $\left\{p_{1}, \cdots, p_{s}\right\}$.

Theorem 3.11. Let $M$ be a module over a Noetherian ring $R$. Let $N$ be a proper submodule of $M$ satisfying the primful property. If $N=\cap_{i=1}^{s} N_{i}$ is a module-reduced quasi-primary decomposition and $N_{i}$ satisfies the primeful property, $1 \leq i \leq s$, such that $\operatorname{radN}=\cap_{i=1}^{s} \operatorname{rad} N_{i}$. If $p_{j}=\sqrt{\left(N_{j}: M\right)}$ is a minimal element of $\left\{p_{1}, \cdots, p_{s}\right\}$, then $\operatorname{rad} N_{j}$ is uniquely determined by $N$.

\section{QUASI-PRIMARY SUBMODULES OF MULTIPLICATION MODULES}

Let $M$ be a multiplication $R$-module. If $p$ is a prime ideal containing $\operatorname{ann}(M)$, then $(p M: M)=p[7$, Lemma 2.10]. In particular a proper submodule $p M$ is a prime submodule of $M$ if and only if $p$ is a prime ideal containing $\operatorname{ann}(M)$ [7, Corollary 2.11]. Now we have the following result:

2.1. Lemma. Let $R$ be a ring and $I$ an ideal of $R$. Let $M$ be a multiplication $R$-module. If $I M$ satisfies the primeful property, then so does $\sqrt{I} M$. In this case $\sqrt{(I M: M)}=$ $\sqrt{(\sqrt{I} M: M)}$.

Proof. Let $p$ be a prime ideal containing $(\sqrt{I} M: M)$. Since $I M$ satisfies the primeful property, there exists a prime submodule $P$ containing $I M$ such that $(P: M)=p$. By $[7$, Corollary 2.11], $P=p^{\prime} M$ for some prime ideal $p^{\prime}$ containing ann $(M)$. Since $I M \subseteq p^{\prime} M$, by [7, Lemma 2.10] $I \subseteq p^{\prime}$. Hence $\sqrt{I} M \subseteq P$, as required. Also the similar argument follows that $\operatorname{rad}(I M)=\operatorname{rad}(\sqrt{I} M)$ and so we have the second part.

2.2. Theorem. Let $N$ be a submodule of a multiplication $R$-module $M$ which satisfies the primeful property. Then the following statements are equivalent:

(i) $N$ is a quasi-primary submodule of $M$;

(ii) $(N: M)$ is a quasi-primary ideal of $R$;

(iii) $\operatorname{rad} N$ is a prime submodule of $M$;

(iv) $N=q M$ for some quasi-primary ideal $q$ of $R$ with ann $(M) \subseteq q$.

Proof. (i) $\Rightarrow$ (ii) is clear, since $\sqrt{(N: M)}=(\operatorname{rad} N: M)$.

(ii) $\Rightarrow$ (iii). It is easy to check that $\operatorname{rad} N$ is a proper submodule of $M$, since $N$ satisfies the primeful property. Now the proof is completed by [7, Corollary 2.11 and Theorem $2.12]$.

(iii) $\Rightarrow$ (i) is obtained by a direct application of the definition of quasi-primary submodules.

(ii) $\Rightarrow$ (iv) is clear.

(iv) $\Rightarrow$ (iii). Let $q$ be a quasi-primary ideal of $R$ containing $(0: M)$ and $N=q M$. By [7, Theorem 2.12] and Lemma 2.1, we have $\operatorname{rad} N=\sqrt{(N: M)} M=\sqrt{(q M: M)} M=$ $\sqrt{(\sqrt{q} M: M)} M=\sqrt{\sqrt{q}} M=\sqrt{q} M$. Thus by [7, Corollary 2.11], $\operatorname{radN}$ is a prime submodule of $M$. 
2.3. Example. Let $M$ be a finitely generated faithful multiplication $R$-module (for example $M$ can be considered as a non-zero ideal of a principal ideal domain $R$ ). Then for each ideal $I$ of $R,(I M: M)=I$ [7, Theorem 3.1]. Thus if $q$ is a quasi-primary ideal of $R$ which is not primary, then $q M$ is a quasi-primary submodule of $M$ which is not primary (see Theorem 2.2 above.)

2.4. Proposition. Let $M$ be a non-zero multiplication $R$-module. If ann $(x)=0$ for some $x \in M$, then every submodule of $M$ satisfies the primeful property.

Proof. Assume $N$ is a submodule of $M$ and $p$ a prime ideal of $R$ containing $(N: M)$. It suffices to show that $p M$ is a prime submodule of $M$. By [7, Corollary 2.11], we must prove that $p M \neq M$. Assume on the contrary that $p M=M$. Suppose $x \in M$ and $\operatorname{ann}(x)=0$. Since $M$ is multiplication, there exists an ideal $J$ of $R$ such that $R x=J M$. Thus $R x=J M=J p M=p J M=p x$ and so $1-r \in \operatorname{ann}(x)$ for some $r \in p$, a contradiction.

It is well-known that if $M$ is a finitely generated multiplication $R$-module, then $M$ is weak cancellation, i.e. $I M \subseteq J M$, for ideals $I, J$ of $R$, implies $I \subseteq J+\operatorname{ann}(M)$ ([1, Theorem 3] and [22, Corollary to Theorem 9]). By combining this fact and Theorem 2.2, we have the following immediate result.

2.5. Corollary. Let $N$ be a submodule of a finitely generated multiplication $R$-module M. Then

(i) $N$ is a minimal quasi-primary submodule of $M$ if and only if there exists a minimal quasi-primary ideal $\mathrm{q}$ of $R$ containing ann $(M)$ such that $N=\mathrm{q} M \neq M$.

(ii) Every quasi-primary submodule of $M$ contains a minimal quasi-primary submodule.

Proof. (i) is clear.

(ii). It suffices to show that every quasi-primary ideal of $R$ contains a minimal quasiprimary ideal. Let $q$ be a quasi-primary ideal of $R$ and $\Lambda=\{\mathfrak{q}: \mathfrak{q}$ is a quasi-primary ideal of $R$ with $\mathfrak{q} \subseteq \mathrm{q}\}$. Since $\mathrm{q} \in \Lambda$, we have $\Lambda \neq \emptyset$. We define a partially order by reverse inclusion, that is, for $\mathfrak{q}_{\mathfrak{i}}, \mathfrak{q}_{\mathfrak{j}} \in \Lambda, \mathfrak{q}_{\mathfrak{i}} \preceq \mathfrak{q}_{\mathfrak{j}}$ if and only if $\mathfrak{q}_{\mathfrak{i}} \supseteq \mathfrak{q}_{\mathfrak{j}}$, so that a maximal member of this partially ordered set is just a minimal member of $\Lambda$ with respect to inclusion. Let $\Omega$ be a non-empty subset of $\Lambda$ which is totally ordered with respect to the above partial order. It is easy to verify that $Q=\cap_{\mathfrak{q} \in \Omega} \mathfrak{q}$ is an upper bound for $\Omega$ in $\Lambda$. Now Zorn's lemma completes the proof.

In [7, Corollary 1.7], it has shown that if $M$ is a multiplication module, then $\cap_{\lambda \in \Lambda}\left(I_{\lambda} M\right)=$ $\left(\cap_{\lambda \in \Lambda}\left[I_{\lambda}+\right.\right.$ ann $\left.\left.M\right]\right) M$ for every non-empty collection of ideals $I_{\lambda}(\lambda \in \Lambda)$ of $R$. Using this fact, we have the following result:

2.6. Corollary. Let $M$ be a multiplication $R$-module and $N$ a submodule of $M$. Let $N_{i}$ $(1 \leq i \leq s)$ be a collection of submodules of $M$ satisfying the primeful property. Then the following statements are equivalent:

(i) $(N: M)=\left(N_{1}: M\right) \cap \cdots \cap\left(N_{s}: M\right)$ is a reduced quasi-primary decomposition of $I$;

(ii) $N=N_{1} \cap \cdots \cap N_{s}$ is a reduced quasi-primary decomposition of $N$;

(iii) $N=N_{1} \cap \cdots \cap N_{s}$ is a module-reduced quasi-primary decomposition of $N$.

2.7. Corollary. Let $I$ be an ideal of $R$ containing ann $(M)$. Let $M$ be a multiplication $R$-module. If $I=q_{1} \cap \cdots \cap q_{s}$ is a reduced quasi-primary decomposition of $I$, then $I M=q_{1} M \cap \cdots \cap q_{s} M$ is a reduced and module-reduced quasi-primary decomposition of $I M$. 
The following is an immediate consequence of Theorem 2.2 and [9, Theorem 1].

2.8. Corollary. Let $M$ be a multiplication $R$-module and $N$ a submodule $M$. Let $N_{i}=$ $q_{i} M,(1 \leq i \leq s)$ be a collection of quasi-primary submodules of $M$ satisfying the primeful property. Then $N_{1} \cap \cdots \cap N_{s}$ is a quasi-primary submodule of $M$ if and only if among the prime ideals $\sqrt{\left(N_{i}: M\right)}$ there is a $\sqrt{\left(N_{k}: M\right)}$ such that $\sqrt{\left(N_{k}: M\right)} \subseteq \sqrt{\left(N_{i}: M\right)}$.

Recall that a representation $N=N_{1} \cap N_{2} \cap \cdots \cap N_{s}$ of a submodule $N$ of an $R$ module $M$ is shortest, if none of the $N_{i}$ can be omitted and none of the intersection $\left(N_{i_{1}}: M\right) \cap\left(N_{i_{2}}: M\right) \cap \cdots \cap\left(N_{i_{t}}: M\right)(t>1)$ is a quasi-primary ideal.

2.9. Proposition. Let $M$ be a multiplication $R$-module and $N$ a submodule $M$. Let $N_{i}=q_{i} M(1 \leq i \leq s)$ be a collection of submodules of $M$ satisfying the primeful property. Then every quasi-primary decomposition $N=N_{1} \cap N_{2} \cap \cdots \cap N_{s}$ has a shortest quasiprimary decomposition.

Proof. First we omit every superfluous term $N_{i}$. Second, assume there exist submodules $N_{i_{1}}, N_{i_{2}}, \cdots, N_{i_{t}}$ such that $\sqrt{\left(N_{i_{1}}: M\right)} \subseteq \sqrt{\left(N_{i_{2}}: M\right)} \subseteq \cdots \subseteq \sqrt{\left(N_{i_{t}}: M\right)}$. Put $N_{i}^{\prime}=$ $N_{i_{1}} \cap N_{i_{2}} \cap \cdots \cap N_{i_{t}}$. Then by Corollary 2.8, $N_{i}^{\prime}$ is a quasi-primary submodule of $M$. Thus $N=N_{1}^{\prime} \cap N_{2}^{\prime} \cap \cdots \cap N_{r}^{\prime}$ is a shortest quasi-primary decomposition of $N$.

2.10. Corollary. Let $M$ be a multiplication module with a submodule $N$. If $N=N_{1} \cap$ $N_{2} \cap \cdots \cap N_{s}$ is a shortest quasi-primary decomposition such that each $N_{i}(1 \leq i \leq s)$ satisfies the primeful property, then all the prime ideals belonging to the quasi-primary submodules which occur in a shortest quasi-primary decomposition of $N$ are isolated.

2.11. Proposition. Let $M$ be a multiplication $R$-module and $N$ a submodule of $M$. Let $N_{i}(1 \leq i \leq s)$ be a collection of submodules of $M$ satisfying the primeful property. If $N=N_{1} \cap N_{2} \cap \cdots \cap N_{s}$ is a shortest quasi-primary decomposition, then it is a reduced and module-reduced quasi-primary decomposition of $N$.

Proof. It is clear that the ideals $\sqrt{\left(N_{i}: M\right)}$ are prime for every $i(1 \leq i \leq t)$. Assume, on the contrary, there exists $j \neq i$ such that $\sqrt{\left(N_{j}: M\right)}=\sqrt{\left(N_{i}: M\right)}$. Then $\left(N_{i}\right.$ : $M) \cap\left(N_{j}: M\right)$ is a quasi-primary ideal of $R$, since $\sqrt{\left(N_{i} \cap N_{j}: M\right)}=\sqrt{\left(N_{i}: M\right)}$ is a prime ideal of $R$, a contradiction. Therefor $N=N_{1} \cap N_{2} \cap \cdots \cap N_{t}$ is a reduced quasiprimary decomposition and by Corollary 2.6 is also a module-reduced quasi-primary decomposition.

In general, the converse of the above proposition is not true. For instance, let $R=$ $K[x, y]$ be the ring of polynomials in $x, y$ with coefficients in a field $K$. Consider the ideal $I=\left(x^{2} y, x y^{2}\right)$ of $R$. It is clear that $\operatorname{rad} I=(x y)$ is not a prime ideal and so $I$ is not quasi-primary. $I=(x) \cap(y) \cap\left(x^{2}, y^{2}\right)$ is a reduced quasi-primary decomposition that is not shortest [9, p. 181].

The following is an immediate result of Proposition 2.6 and Proposition 2.11.

2.12. Corollary. Let $M$ be a multiplication $R$-module and $N$ a submodule of $M$. Let $N_{i}$ $(1 \leq i \leq s)$ be a collection of submodules of $M$ satisfying the primeful property. If $N$ has a quasi-primary decomposition, then it has both reduced and module-reduced quasi-primary decompositions.

2.13. Theorem. Let $M$ be a multiplication $R$-module and $N$ a submodule of $M$. Let $N_{i}=q_{i} M,(1 \leq i \leq s)$ be a collection of submodules of $M$ satisfying the primeful property. Then $N=N_{1} \cap N_{2} \cap \ldots \cap N_{s}$ is a shortest quasi-primary decomposition of $N$ if and only if $(N: M)=\left(N_{1}: M\right) \cap\left(N_{2}: M\right) \cap \ldots \cap\left(N_{s}: M\right)$ is a shortest quasi-primary decomposition of the ideal $(N: M)$. 
Proof. $\Rightarrow)$ Assume, on the contrary, that $(N: M)=\left(N_{1}: M\right) \cap\left(N_{2}: M\right) \cap \cdots \cap\left(N_{s}\right.$ : $M)$ is not shortest. Then either $\left(N_{t}: M\right)$ may be omitted for some $1 \leq t \leq s$ or $\left(N_{i_{1}}: M\right) \cap\left(N_{i_{2}}: M\right) \cap \cdots \cap\left(N_{i_{r}}: M\right)$ is a quasi-primary ideal for some $r>1$. Firstly, assume $\left(N_{t}: M\right) \supseteq\left(N_{1}: M\right) \cap \cdots \cap\left(N_{t-1}: M\right) \cap\left(N_{t+1}: M\right) \cap \cdots \cap\left(N_{s}: M\right)$. Therefor $\sqrt{\left(N_{t}: M\right)} \supseteq \sqrt{\left(N_{1}: M\right)} \cap \cdots \cap \sqrt{\left(N_{t-1}: M\right)} \cap \sqrt{\left(N_{t+1}: M\right)} \cap \cdots \cap \sqrt{\left(N_{m}: M\right)}$. Since $\sqrt{\left(N_{t}: M\right)}$ is a prime ideal, there exists $k \neq t$ such that $\sqrt{\left(N_{k}: M\right)} \subseteq \sqrt{\left(N_{t}: M\right)}$. Now Corollary 2.10 shows that $\sqrt{\left(N_{k}: M\right)}=\sqrt{\left(N_{t}: M\right)}$. Thus $N=N_{1} \cap N_{2} \cap \ldots \cap N_{s}$ is not a reduced quasi-primary decomposition, which contradicts the Proposition 2.11. Secondly, if $\left(N_{i_{1}}: M\right) \cap\left(N_{i_{2}}: M\right) \cap \cdots \cap\left(N_{i_{r}}: M\right)$ is a quasi-primary ideal for some $r>1$,

then there is a minimal prime ideal $\sqrt{\left(N_{i_{k}}: M\right)}$ among the prime ideals $\sqrt{\left(N_{i_{j}}: M\right)}$ $(1 \leq j \leq r)$, which contradicts the Corollary 2.10 .

$\Leftarrow)$ Suppose $(N: M)=\left(N_{1}: M\right) \cap\left(N_{2}: M\right) \cap \ldots \cap\left(N_{s}: M\right)$ is a shortest quasi-primary decomposition of the ideal $(N: M)$ in $R$. Multiplying by $M$, we get $N=N_{1} \cap N_{2} \cap \ldots \cap N_{s}$. It is easy to check that the above representation is a shortest quasi-primary decomposition of $N$.

2.14. Theorem. Let $M$ be a multiplication $R$-module and $N$ a submodule of $M$. Let $N=N_{1} \cap N_{2} \cap \cdots \cap N_{s}=N_{1}^{\prime} \cap N_{2}^{\prime} \cap \cdots \cap N_{t}^{\prime}$ be two reduced quasi-primary decompositions of $N$ as intersection of quasi-primary submodules satisfying the primeful property. Then $s=t$ and the prime ideals $p_{i}=\sqrt{\left(N_{i}: M\right)}$ must be, without regard to their order, identical to the prime ideals $p_{j}^{\prime}=\sqrt{\left(N_{j}^{\prime}: M\right)}$.

Proof. Let $N=N_{1} \cap N_{2} \cap \cdots \cap N_{s}=N_{1}^{\prime} \cap N_{2}^{\prime} \cap \cdots \cap N_{t}^{\prime}$ be two shortest quasi-primary decompositions of $N$. By Theorem 2.13, we have two shortest quasi-primary decompositions $(N: M)=\left(N_{1}: M\right) \cap\left(N_{2}: M\right) \cap \cdots \cap\left(N_{s}: M\right)=\left(N_{1}^{\prime}: M\right) \cap\left(N_{2}^{\prime}: M\right) \cap \cdots \cap\left(N_{t}^{\prime}: M\right)$ of the ideal $(N: M)$. Now the proof is completed by [9, Theorem 6$]$.

2.15. Proposition. Let $N$ and $K$ be quasi-primary submodules of a multiplication $R$ module $M$ satisfying the primeful property. Then $N \cap K$ is quasi-primary if and only if $\operatorname{rad} N \subseteq \operatorname{radK}$ or $\operatorname{radK} \subseteq \operatorname{rad} N$

Proof. Since $N \cap K$ is a quasi-primary submodule, $\sqrt{(N \cap K: M)}=\sqrt{(N: M)} \cap$ $\sqrt{(K: M)}$ is a prime ideal of $R$ and so $\sqrt{(N: M)} \subseteq \sqrt{(K: M)}$ or $\sqrt{(K: M)} \subseteq$ $\sqrt{(N: M)}$. Equivalently $(\operatorname{rad} N: M) \subseteq(\operatorname{rad} K: M)$ or $(\operatorname{rad} K: M) \subseteq(\operatorname{rad} N: M)$. Therefore $\operatorname{radN} \subseteq \operatorname{radK}$ or $\operatorname{radK} \subseteq \operatorname{radN}$, since $M$ is a multiplication module. The reverse argument implies that $(N \cap K: M)$ is a quasi-primary ideal and so by Theorem $2.2, N \cap K$ is a quasi-primary submodule of $M$.

\section{QUASI-PRIMARY DECOMPOSITION OF SUBMODULES OF MODULES OVER NOETHERIAN RINGS}

In [6, Theorem 3.10], it has been shown that every proper submodule of a Noetherian module has a primary decomposition and so a fortiori quasi-primary decomposition. In particular, every submodule of finitely generated modules or faithful multiplication modules over Noetherian rings has a quasi-primary decomposition [7, p.764]. This gives rise to the question: is there a submodule of a module which has a quasi-primary decomposition, but has not any primary decomposition. Let us now present positive answer to this question below.

3.1. Example. Since the set of ideals of a valuation domain is totally ordered under inclusion, we conclude that every proper ideal of a valuation domain is quasi-primary [11, Theorem 5.10]. On the other hand, it is proved that for a local domain $R$, every 
proper ideal of $R$ is primary if and only if $\operatorname{dim} R=1$ [4, Theorem2.4]. Now let $R$ be a valuation domain with $\operatorname{dim} R>1$. Then there exists a quasi-primary ideal $q$ of $R$ which is not primary. Now if $q=q_{1} \cap q_{2} \cap \cdots \cap q_{n}$ is a reduced primary decomposition of $q$, then there is $1 \leq j \leq n$ such that $q_{j} \subseteq \sqrt{q} \subseteq \sqrt{q_{j}}$. Thus $\sqrt{q_{j}}$ is a minimal element of the set $\left\{\sqrt{q_{1}}, \sqrt{q_{2}}, \cdots, \sqrt{q_{n}}\right\}$. We claim that $q_{j}$ is minimal among the ideals $q_{1}, q_{2}, \cdots, q_{n}$ and so $q=q_{j}$. This contradicts the choice of $q$. Let $q_{i} \subseteq q_{j}$ for some $i \neq j$. By minimality of $\sqrt{q_{j}}$ we must have $\sqrt{q_{i}}=\sqrt{q_{j}}$, which contradicts the fact that $q=q_{1} \cap q_{2} \cap \cdots \cap q_{n}$ is a reduced primary decomposition of $q$. Thus $q_{i} \nsubseteq q_{j}$ for every $i \neq j$. Now since the set of ideals of $R$ is totally ordered under inclusion, we must have $q_{j} \subseteq q_{i}$ for every $i \neq j$, as required.

It has been shown that a reduced primary decomposition is unique in the sense of the set of prime ideals belonging to primary submodules of two primary decompositions are the same and the set of primary submodules with isolated associated primes are also identical [6, Theorem 3.10]. In this section we study quasi-primary submodules of modules over Noetherian rings. In particular, we give some uniqueness theorems for reduced and module-reduced quasi-primary decomposition (Theorem 3.6, Theorem 3.8 and Theorem 3.12).

3.2. Lemma. Let $R$ be a Noetherian ring and $N$ a p-quasi-primary submodule of an $R$-module $M$. Then there exists a positive integer $n$ such that $p^{n} \subseteq(N: M)$.

Proof. Taking $p=\left(r_{1}, \cdots, r_{t}\right)$. For each generator $r_{i}$, there is a positive integer $n_{i}$ such that $r_{i}^{n_{i}} \in(N: M)$. Let $n$ has the value $n=\sum_{i=1}^{t}\left(n_{i}-1\right)+1$. Now $p^{n}$ is generated by monomials $r_{1}^{m_{1}} \cdots r_{t}^{m_{t}}$ with $\sum_{j=1}^{t} m_{j}=n$, because at least for one of the subscripts $j$ we have $s_{j} \geq n$. Hence $p^{n} \subseteq(N: M)$.

Since a faithful multiplication module $M$ over a Noetherian $\operatorname{ring} R$ is Noetherian ([7, p.764]), then every submodule of $M$ satisfies the primeful property. Thus we can replace "satisfying the primeful property" for these submodules of $M$ with "faithfulness" for $M$ in Theorem 3.3 and and Theorem 3.5.

3.3. Theorem. Let $R$ be a Noetherian ring and $M$ a multiplication $R$-module. Let $N$ be a submodule of $M$ which satisfies the primeful property. Then $N$ is quasi-primary if and only if there exists a unique prime ideal $p$ of $R$ such that $p^{t} \subseteq(N: M) \subseteq p$ for some positive integer $t$.

Proof. $(\Rightarrow)$ By Theorem 2.2, $(N: M)$ is a quasi primary ideal. If $p=\sqrt{(N: M)}$, then by Lemma $3.2 p^{t} \subseteq(N: M) \subseteq p$ for some positive integer $t$. If $p^{\prime}$ is a prime ideal of $R$ and $p^{\prime s} \subseteq(N: M) \subseteq p^{\prime}$, then $p^{\prime}=\sqrt{(N: M)}=p$.

$(\Leftarrow)$ It is clear that $(N: M)$ is quasi-primary ideal. Now the proof is completed by Theorem 2.2.

3.4. Lemma. Let $M$ be a multiplication $R$-module and $N_{1}$ a submodule of $M$. Let $\mathrm{N}_{2}$ be a quasi-primary submodule of $M$ satisfying the primeful property such that $p=$ $\sqrt{\left(N_{1}: M\right)}=\sqrt{\left(N_{2}: M\right)}$ and $N_{1} \subseteq N \subseteq N_{2}$. Then $N$ is a p-quasi-primary submodule of $M$.

Proof. It is clear that $\sqrt{\left(N_{1}: M\right)}=\sqrt{(N: M)}=\sqrt{\left(N_{2}: M\right)}=p$ and so $(N: M)$ is a $p$-quasi-primary ideal of $R$. Now if $\mathrm{p}$ is a prime ideal containing $(N: M)$, then $\left(N_{2}: M\right) \subseteq$ p. Since $N_{2}$ satisfies the primeful property, there exists a prime submodule $P$ containing $N_{2}$ and so $N$ such that $(P: M)=$ p. Thus $N$ satisfies the primeful property. Now by Theorem 2.2, $N$ is a $p$-quasi-primary submodule of $M$. 
3.5. Theorem. Let $R$ be a Noetherian ring and $M$ a multiplication $R$-module. Let $N_{i}$ $(1 \leq i \leq t)$ be a collection of quasi-primary submodules of $M$ with $\sqrt{\left(N_{i}: M\right)}=p_{i}$. If $N_{1}$ satisfies the primeful property and $p_{1} \subseteq p_{i}$ for each $1 \leq i \leq t$, then $N=\left(\Pi_{i=1}^{t}\left(N_{i}: M\right)\right) M$ is also $p_{1}$-quasi-primary.

Proof. Since $R$ is a Noetherian ring there are positive integers $s_{i}(1 \leq i \leq t)$ such that $p_{1}^{s_{1}+s_{2} \cdots+s_{t}} M \subseteq p_{1}^{s_{1}} p_{2}^{s_{2}} \cdots p_{t}^{s_{t}} M \subseteq\left(N_{1}: M\right)\left(N_{2}: M\right) \cdots\left(N_{t}: M\right) M \subseteq p_{1} p_{2} \cdots p_{t} M \subseteq$ $p_{1} M$. Thus

$$
p_{1} \subseteq \sqrt{\left(p_{1}^{s_{1}+s_{2} \cdots+s_{t}} M: M\right)} \subseteq \sqrt{\left(p_{1} p_{2} \cdots p_{t} M: M\right)} \subseteq p_{1}
$$

and so $\sqrt{\left(p_{1} p_{2} \cdots p_{t} M: M\right)}=p_{1}$. Now by a similar consideration of Lemma 3.4, it can be shown that $p_{1} p_{2} \cdots p_{t} M$ satisfies the primeful property. Hence by Theorem 2.2, $N=\left(\Pi_{i=1}^{t}\left(N_{i}: M\right)\right) M$ is $p_{1}$-quasi-primary.

3.6. Theorem. Let $R$ be a Noetherian ring and $M$ an $R$-module. Let $N$ be a submodule of $M$ such that $N=N_{1} \cap N_{2} \cap \cdots \cap N_{s}=N_{1}^{\prime} \cap N_{2}^{\prime} \cap \cdots \cap N_{t}^{\prime}$ be two reduced quasi-primary decompositions of $N$ where $N_{i}$ (resp. $N_{j}^{\prime}$ ) is $\mathrm{p}_{i}$-quasi-primary (resp. $\mathfrak{p}_{j}$-quasi-primary). Then $s=t$ and (after reordering if necessary) $\mathrm{p}_{i}=\mathfrak{p}_{i}$ and $\operatorname{rad} N_{i}=\operatorname{rad} N_{i}^{\prime}$ for $1 \leq i \leq s$.

Proof. Without loss of generality we may assume that $\mathfrak{p}_{1}$ is one of the minimal elements of the set $\left\{\mathrm{p}_{1}, \cdots \mathrm{p}_{s}, \mathfrak{p}_{1}, \cdots \mathfrak{p}_{t}\right\}$. Since $N_{1}$ is $\mathrm{p}_{1}$-quasi-primary, there exists a positive integer $t$ such that $\mathrm{p}_{1}^{t} M \subseteq N_{1}$ and hence

$$
\mathrm{p}_{1}^{t}\left(N_{2} \cap N_{3} \cap \cdots \cap N_{s}\right) \subseteq N=N_{1}^{\prime} \cap N_{2}^{\prime} \cap \cdots \cap N_{t}^{\prime} .
$$

If $N_{2} \cap N_{3} \cap \cdots \cap N_{s} \subseteq \operatorname{rad} N_{1}^{\prime}$, then we have $\cap_{i=2}^{s} \mathrm{p}_{i} \subseteq \mathfrak{p}_{1}$ and so $\mathrm{p}_{i} \subseteq \mathfrak{p}_{1}$ for some $2 \leq i \leq s$. Thus by assumption $\mathrm{p}_{i}=\mathfrak{p}_{1}$ for some $2 \leq i \leq s$. In the other case, suppose $N_{2} \cap N_{3} \cap \cdots \cap N_{s} \nsubseteq \operatorname{rad} N_{1}^{\prime}$. Since $N_{1}^{\prime}$ is quasi-primary, we have $\mathrm{p}_{1}^{t} \subseteq \mathfrak{p}_{1}$ and hence $\mathrm{p}_{1} \subseteq \mathfrak{p}_{1}$. Now by minimality of $\mathfrak{p}_{1}$, we conclude that $\mathrm{p}_{1}=\mathfrak{p}_{1}$. Since $\left\{\mathrm{p}_{1}, \mathrm{p}_{2} \cdots, \mathrm{p}_{s}\right\}$ and $\left\{\mathfrak{p}_{1}, \mathfrak{p}_{2} \cdots, \mathfrak{p}_{t}\right\}$ are sets of distinct prime ideals, with a similar argument we have $s=t$ and $\mathrm{p}_{i}=\mathfrak{p}_{i}$ for $1 \leq i \leq s$.

For the second part, since $\mathrm{p}_{i}$ are all distinct, there exists $r_{i} \in \mathrm{p}_{i} \backslash \mathrm{p}_{1}$ for each $2 \leq i \leq s$. Then $r=r_{2} r_{3} \cdots r_{s} \in \mathrm{p}_{i}$ for $i>1$, but $r \notin \mathrm{p}_{1}$. Since $N_{i}\left(\right.$ resp. $\left.N_{i}^{\prime}\right)$ is $\mathrm{p}_{i}$-quasi-primary, there exists an integer $n_{i}\left(\right.$ resp. $\left.m_{i}\right)$ such that $r^{n_{i}} \in\left(N_{i}: M\right)\left(\operatorname{resp} . r^{m_{i}} \in\left(N_{i}^{\prime}: M\right)\right)$ for each $2 \leq i \leq s$. Let $n=\max \left\{n_{2}, \cdots, n_{s}, m_{2} \cdots, m_{s}\right\}$. Then $r^{n} \in\left(N_{i}: M\right)$ and $r^{n} \in\left(N_{i}^{\prime}: M\right)$ for each $2 \leq i \leq s$. Now if $x \in N_{1}$, then $r^{n} x \in N$ whence $r^{n} x \in N_{1}^{\prime}$. It follows from the definition that $x \in \operatorname{rad} N_{1}^{\prime}$. Therefore $N_{1} \subseteq \operatorname{rad} N_{1}^{\prime}$. A similar argument shows that $N_{1}^{\prime} \subseteq \operatorname{rad} N_{1}$ and hence $\operatorname{rad} N_{1}=\operatorname{rad} N_{1}^{\prime}$.

3.7. Lemma. Let $M$ be an $R$-module. If $\left\{N_{i}: 1 \leq i \leq t\right\}$ is a finite collection of submodules of $M$ which satisfy the primeful property, then so does $\cap_{i=1}^{t} N_{i}$.

Proof. Clear.

3.8. Theorem. Let $N$ be a proper submodule of a module $M$ over a Noetherian ring $R$. If $N=\cap_{i=1}^{t} N_{i}$ is a module-reduced quasi-primary decomposition and $N_{i}(1 \leq i \leq t)$ satisfies the primeful property such that $\operatorname{rad} N=\cap_{i=1}^{t} \operatorname{rad} N_{i}$, then $\operatorname{Ass}(M / \operatorname{rad} N) \subseteq$ $\left\{p_{1}, \cdots, p_{t}\right\} \subseteq \operatorname{Supp}(M / \operatorname{rad} N)$. In particular, Ass $(M / \operatorname{rad} N)=\left\{p_{i_{1}}, p_{i_{2}}, \cdots, p_{i_{s}}\right\}$ where $p_{i_{j}}(1 \leq j \leq s)$ are minimal elements of $\left\{p_{1}, \cdots, p_{t}\right\}$. 
Proof. Let $p$ be an associated prime of $M / \operatorname{rad} N$, so that $p=\operatorname{ann}(x+\operatorname{rad} N), 0 \neq$ $x+\operatorname{rad} N \in M / \operatorname{rad} N$. Renumber the $N_{i}$ so that $x \notin \operatorname{rad} N_{i}$ for $1 \leq i \leq j$ and $x \in \operatorname{rad} N_{i}$ for $j+1 \leq i \leq t$. Since $N_{i}$ is a quasi-primary submodule satisfying the primeful property, $p_{i}=\sqrt{\left(N_{i}: M\right)}$ is a prime ideal of $R(1 \leq i \leq t)$. Since $p_{i}$ is finitely generated, $p_{i}^{n_{i}} M \subseteq$ $N_{i}$ for some $n_{i} \geq 1$. Therefore $\left(\cap_{i=1}^{j} p_{i}^{n_{i}}\right) x \subseteq \cap_{i=1}^{t} \operatorname{rad} N_{i}=\operatorname{rad} N$, so $\cap_{i=1}^{j} p_{i}^{n_{i}} \subseteq \operatorname{ann}(x+$ $\operatorname{rad} N)=p$. Since $p$ is prime, $p_{i} \subseteq p$ for some $i \leq j$. We claim that $p_{i}=p$, so that every associated prime must be one of the $p_{i}$ 's. To verify this, let $r \in p$. Then $r(x+\operatorname{rad} N)=$ $\operatorname{radN}$ and $x \notin \operatorname{rad} N_{i}$ and since $\operatorname{rad} N_{i}$ is prime we have $r \in \sqrt{\left(N_{i}: M\right)}=p_{i}$, as claimed. By [8, Lemma 3.4], $M / \operatorname{rad} N_{i}$ is a primeful $R$-module. Now since $p_{i} \supseteq(\operatorname{radN}: M)$ for each $1 \leq i \leq t$, we have $A s s(M / \operatorname{rad} N) \subseteq\left\{p_{1}, p_{2}, \cdots, p_{t}\right\} \subseteq \operatorname{Supp}(M / \operatorname{radN})$, by [12, Proposition 3.4]. For the second part, we show that minimal elements of $\left\{p_{1}, \cdots, p_{t}\right\}$ are equal to minimal elements of $\operatorname{Supp}(M / \operatorname{radN})$. Let $p_{j}$ be a minimal element of $\left\{p_{1}, \cdots, p_{t}\right\}$ and $p \subseteq p_{j}$ for some $p \in S u p p(M / \operatorname{radN})$. By [8, Lemma 3.4] and Lemma 3.7 $\operatorname{rad} N$ satisfies the primeful property and hence by [12, Proposition 3.4] $p \supseteq(\operatorname{rad} N: M)$. Thus $\cap_{i=1}^{t} p_{i} \subseteq p \subseteq p_{j}$. Since $p$ is prime, there exists $p_{i}(1 \leq i \leq t)$ such that $p_{i} \subseteq p \subseteq p_{j}$ and so $p_{i}=p=p_{j}$, by minimality of $p_{j}$. Now the proof is completed by [20, Theorem $9.39]$.

Noth that, by the proof of Theorem 3.8, the minimal prime ideals of the set $\left\{p_{1}, \cdots, p_{t}\right\}$ are uniquely determined by $N$, as follows.

3.9. Corollary. Let $N$ be a proper submodule of a module $M$ over a Noetherian ring R. Let $N=\cap_{i=1}^{t} N_{i}$ be a module-reduced quasi-primary decomposition and $N_{i}$ satisfies the primeful property, $1 \leq i \leq t$, such that $\operatorname{rad} N=\cap_{i=1}^{t} \operatorname{rad} N_{i}$. Let $p_{i}=\sqrt{\left(N_{i}: M\right)}$ for $1 \leq i \leq t$. Then the minimal primes which occur in the set $\left\{p_{1}, \cdots, p_{t}\right\}$ are uniquely determined by $N$.

3.10. Corollary. Let $N$ be a proper submodule of a module $M$ over a Noetherian ring $R$ which satisfies the primeful property. Then $N$ is p-quasi-primary if and only if $\operatorname{Ass}(M / \operatorname{rad} N)=p$.

3.11. Lemma. Let $M$ be a module over a Noetherian ring $R$, and $N$ a quasi-primary submodule of $M$ satisfying the primeful property with $p=\sqrt{(N: M)}$. Let $p^{\prime}$ be any prime ideal of $R$.

(i) If $p \nsubseteq p^{\prime}$, then $M_{p^{\prime}}=(\operatorname{rad} N)_{p^{\prime}}$.

(ii) If $p \subseteq p^{\prime}$, then $\operatorname{rad} N=f^{-1}\left((\operatorname{rad} N)_{p^{\prime}}\right)$ where $f$ is the mapping $x \mapsto x / 1$ from $M$ into $M_{p^{\prime}}$.

Proof. (i). It is easy to verify that there is a bijection between $A s s_{R_{p^{\prime}}}(M / \operatorname{radN})_{p^{\prime}}$ (which coincide with $\left.A s s_{R_{p^{\prime}}}\left(M_{p^{\prime}} /(\operatorname{rad} N)_{p^{\prime}}\right)\right)$ and the intersection $A s s_{R}(M / \operatorname{rad} N) \cap S$, where $S$ is the set of prime ideals contained in $p^{\prime}$. By Corollary 3.10, there is only one associated prime of $M / \operatorname{radN}$ over $R$, namely $p$, which is not contained in $p^{\prime}$ by hypothesis. Thus $A s s_{R}(M / \operatorname{radN}) \cap S$ is empty, so by [20, Corollary 9.35], $M_{p^{\prime}} /(\operatorname{radN})_{p^{\prime}}=0$, and the result follows.

(ii). As in Corollary 3.10, $A s s_{R}(M / \operatorname{rad} N)=\{p\}$. Since $p \subseteq p^{\prime}$, we have $R \backslash p^{\prime} \subseteq$ $R \backslash p$. By [20, Corollary 9.36], $R \backslash p^{\prime}$ contains no zero-divisors of $M / \operatorname{radN}$, because all such zero-divisors belong to $p$. Thus the natural map $g: x \rightarrow x / 1$ from $M / \operatorname{rad} N$ to $(M / \operatorname{rad} N)_{p^{\prime}} \cong\left(M_{p^{\prime}} /(\operatorname{rad} N)_{p^{\prime}}\right)$ is injective. Assume $x \in f^{-1}\left((\operatorname{rad} N)_{p^{\prime}}\right)$. Then $f(x) \in$ $(\operatorname{rad} N)_{p^{\prime}}$, so $f(x)+(\operatorname{rad} N)_{p^{\prime}}$ is 0 in $M_{p^{\prime}} /(\operatorname{rad} N)_{p^{\prime}}$. By injectivity of the natural map $M / \operatorname{rad} N \rightarrow(M / \operatorname{rad} N)_{p^{\prime}}, x+\operatorname{rad} N$ is 0 in $M / \operatorname{rad} N$, in other words, $x \in \operatorname{rad} N$. Thus $f^{-1}\left((\operatorname{rad} N)_{p^{\prime}}\right) \subseteq \operatorname{rad} N$ and the reverse inclusion is clear. 
3.12. Theorem. Let $N$ be a proper submodule of a module $M$ over a Noetherian ring $R$ satisfying the primeful property. If $N=\cap_{i=1}^{t} N_{i}$ is a module-reduced quasi-primary decomposition and $N_{i}$ satisfies the primeful property, $1 \leq i \leq t$, such that radN= $\cap_{i=1}^{t} r a d N_{i}$. If $p_{j}=\sqrt{\left(N_{j}: M\right)}$ is a minimal element of $\left\{p_{1}, \cdots, p_{t}\right\}$, then $r a d N_{j}$ is uniquely determined by $N$.

Proof. Suppose that $p_{j}$ is minimal, so that $p_{j} \nsupseteq p_{i}, i \neq j$. By Lemma 3.11(i) with $p=p_{i}, p^{\prime}=p_{j}$, we have $\left(\operatorname{rad} N_{i}\right)_{p_{j}}=M_{p_{j}}$ for $i \neq j$. By Lemma 3.11(ii), we have $\operatorname{rad} N_{j}=f^{-1}\left(\left(\operatorname{rad} N_{j}\right)_{p_{j}}\right)$, where $f$ is the natural map from $M$ to $M_{p_{j}}$. Hence we have

$$
\begin{aligned}
(\operatorname{radN})_{p_{j}} & =\left(\operatorname{rad} N_{j}\right)_{p_{j}} \cap\left(\cap_{i \neq j}\left(\operatorname{rad} N_{i}\right)_{p_{j}}\right) \\
& =\left(\operatorname{rad} N_{j}\right)_{p_{j}} \cap M_{p_{j}}=\left(\operatorname{rad} N_{j}\right)_{p_{j}} .
\end{aligned}
$$

Thus $\operatorname{rad} N_{j}=f^{-1}\left(\left(\operatorname{rad} N_{j}\right)_{p_{j}}\right)=f^{-1}\left((\operatorname{radN})_{p_{j}}\right)$ depends only on $N$ and $p_{j}$, and since $p_{j}$ is the minimal prime associated with $N$, it follows that $\operatorname{rad} N_{j}$ depends only on $N$.

Acknowledgements. The author would like to thank the referee/referees for a number of constructive comments and valuable suggestions.

\section{References}

[1] D. D. Anderson, Multiplication Ideals, Multiplication Rings and the Ring R[X]. Canad. J. Math., 28 (1976), 760-768.

[2] M. F. Atiyah, I. G. Macdonald, Introduction to Commutative Algebra, Addison-Wesley publishing company, 1969.

[3] M. Behboodi, On the Prime Radical and Bear's Lower Nilradical of Modules, Acta Math. Hungar. (3) (2009), 293-306.

[4] R. Chaudhuri, A Note on Generalized Primary Rings, Mat. Vesnik 13(28)(1976), 375-377.

[5] J. Dauns, Prime Modules, J. Reine Angew. Math., 298 (1978), 156-181.

[6] D. Eisenbud, Commutative Algebra with a View Toward Algebraic Geometry, SpringerVerlag, 1994.

[7] Z. A. EL-Bast, P. F. Smith, Multiplication Modules, Comm. Algebra, 16(4) (1988), 755-779.

[8] H. Fazaeli Moghimi, M. Samiei, Quasi-primary Submodules Satisfying the Primeful Property I, Hacet. J. Math. Stat., Submitted.

[9] L. Fuchs, On Quasi-primary Ideals, Acta Sci. Math. (Szeged), 11 (1947) 174-183.

[10] J. Jenkins, P. F. Smith, On the Prime Radical of a Module over a Commutative Ring, Comm. Algebra, 20 (1992), 3593-3602.

[11] M. D. Larsen, P. J. McCarthy, Multiplicative Ideal Theory, Academic press, 1971.

[12] C. P. Lu, A Module Whose Prime Spectrum Has the Surjective Natural Map, Houston J. Math., 33(1) (2007),127-143.

[13] C. P. Lu, M-radicals of Submodules in Modules II, Math. Japonica., 35 (1990), 991-1001.

[14] R. McCasland, M. Moore, Prime Submodules. Comm. Algebra, 20 (1992), 1803-1817.

[15] R. L. McCasland, M. E. Moore, P. F. Smith, On the Spectrum of a Module over a Commutative Ring, Comm. Algebra, 25(1) (1997), 79-103.

[16] R. L. McCasland, P. F. Smith, Generalised Associated Primes and Radicals of Submodules, Int. Electron. J. Algebra, 4 (2008), 159-176.

[17] M. E. Moor, S. J. Smith, Prime and Radical Submodules of Modules over Commutative Rings, Comm. Alegbra, 30 (2002), 5073-5064.

[18] R. Naghipour, M. Sedghi, Weakly Associated Primes and Primary Decomposition of Modules over Commutative Rings, Acta Math. Hungar. 110 (1-2) (2006), 1-12.

[19] H. Sharif, Y. Sharifi, S. Namazi, Rings Satisfying the Radical Formula, Acta Math. Hungar., 71(1-2) (1996), 103-108.

[20] R. Y. Sharp, Steps in Commutative Algebra, Cambridge University Press, Cambridge, 1990.

[21] P. F. Smith, Uniqueness of Primary Decompositions, Turk. J. Math. 27 (2003), 425-434.

[22] P. F. Smith, Primary Modules over Commutative Rings, Comm. Algebra, 43 (2001), 103111. 
[23] P. F. Smith, Some Remarks on Multiplication Modules, Arch. der Math., 50 (1988), 223-235.

[24] A. Soleyman Jahan, Prime Filtrations and Primary Decompositions of Modules, Comm. Algebra, 39 (2011), 116-124.

[25] D. Pusat-Yilmaz, P.F. Smith, Radicals of Submodules of Free Modules, Comm. Algebra, 27(5) (1999), 2253-2266.

[26] D. Pusat-Yilmaz, P. F. Smith, Modules Which Satisfy the Radical Formula, Acta Math. Hungar. 95 (1-2) (2002), 155-167. 
